\title{
Flower Microbiology: Old concept but new approach
}

\author{
Ahtesham Ahmad Shad ${ }^{1 *}$ and Wajahat Ahmed Shad ${ }^{2}$ \\ ${ }^{1}$ Institute of Microbiology, University of Agriculture Faisalabad, Pakistan \\ ${ }^{2}$ Department of Biochemistry, University of Agriculture Faisalabad, Pakistan \\ *Corresponding author: Ahtesham Ahmad Shad, Institute of Microbiology, University of Agriculture Faisalabad, Pakistan \\ To Cite This Article: Ahtesham Ahmad Shad. Flower Microbiology: Old concept but new approach. Am J Biomed Sci \& Res. 2019 - 5(4). AJBSR. \\ MS.ID.000933. DOI: 10.34297/AJBSR.2019.05.000933.
}

Received: 留September 23, 2019; Published: 紫 September 30, 2019

\section{Opinion}

Since the ancient times and till now, flowers have been considered as a significant entity and nature's gift. Flowers imparted several key roles in art, religion, health and ornamental arena. Like other recent innovative studies, exploring human microbiota especially the gut microbiome the plant microbiota or plant microbiome has also gained a grained vision to unfold the hidden mysteries of biological world. Moreover, symbiosis of plants with the microbes are also an interesting debate nowadays $[1,2]$. Although it was shown in 1800's that flowers inhabited with microbes but the ecological relationship between them remained undiscovered $[3,4]$. Aforementioned already a long time in history, when flowers and other parts of plants studied but the relation of plant in respect to microbe was first investigated by Lorenz Hiltner dated back to 1904 [5]. The gradual understanding of complex associations and ecological role between plants and respective species of microbes opened unknown concepts. In addition to this, a term "phyllosphere" was coined by F.T Last in 1955 further stretching the chain of information, calling the aboveground portion of plants as the habitat for microbial communities [6].

The studies exploring to the new habitats of microorganisms in plants come up with the rhizosphere (roots), the phyllosphere (leaves), the caulosphere (stem), the anthosphere (Flowers), the carposphere (fruits), and the endosphere (indigenous or inner all parts). Here, we want to introduce a new term for studying flowers in relationship with microorganisms that is "Flower Microbiology". As anthosphere is a term above mentioned dedicated towards the flowers and microbes, but in general, to the best of our knowledge no such term or word has been used previously to describe in simple terms as "Flower Microbiology". We believe that need of time also demanded this field to pay attention and awareness as well because of the increasing burden of Antimicrobial resistance (AMR) day by day. No doubt, some synthesized literature and piece of data we have today but we cannot neglect scarcity in the available information and the existing gaps between flowers and microbes.
The most significant among is the edible flower microbiota or the edible flower microbiome.

So, Flower microbiology aim to give an idea to better understanding and classifying the flower microbes. Flower microbiology can give a way of taxonomical arrangements of groups. For example, edible flowers, non-edible flowers, medicinal flowers etc. This field is no doubt has been in negligence so far, due to lack of knowledge and insufficient data but we believe strongly that the "Flower Microbiology" arena could have been a strong potential to limit or eradicate AMR providing us as an alternative approach to drugs. This has proven by several reports that flowers have been a potent agent of phenolics and antioxidants fulfilling the nutritional need and showing medicinal properties as well by eliminating the hazardous substances during endogenous metabolic processes such as reactice oxygen species (ROS) [7]. Moreover, antibacterial activities of many flowers has also provide a way to control the AMR. Antimicrobial resistance has s strong relation with the origin of drugs as shown in Figure 1.

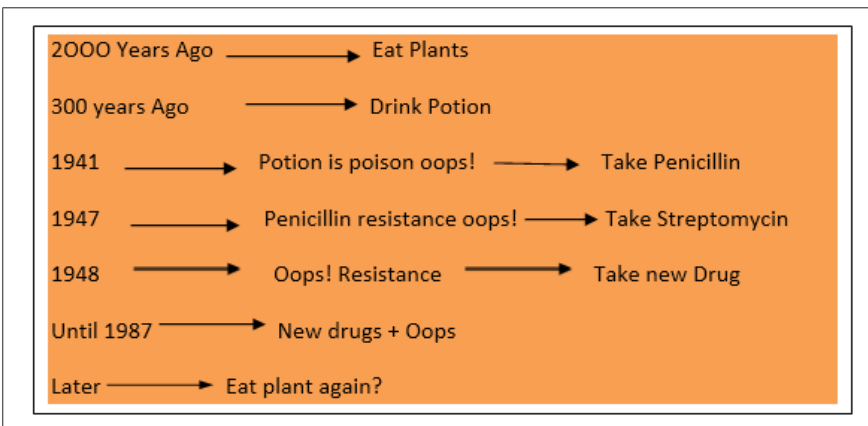

Figure 1: Schematical representation of historical relationship between plants, drugs and resistance.

In a nutshell, the term "Flower Microbiology", convey an easy approach towards the better understanding and keeping differentiation between other related areas of microbiology. This can also lead us to further minute disciplines within it and much more. 


\section{Conclusion}

We recommend the general and broader use of the field of flowers and respective microbes with the term "Flower Microbiology". This may help in easy illustration and classifying the known and as well as the unknown concepts about the ecological relationship between microbes and flowers. Moreover, this can be helpful in uprising the importance of flowers and their microbiota as well because of the simple meanings and can be studied as newly synthesized science discipline.

\section{Reference}

1. Berg G, Mahnert A, Moissl Eichinger C (2014) Beneficial effects of plant-associated microbes on indoor microbiomes and human health? Front Microbiol 5: 15.
2. Mendes R, Garbeva P, Raaijmakers JM (2013) The rhizosphere microbiome: significance of plant beneficial, plant pathogenic, and human pathogenic microorganisms. FEMS Microbiol Rev 37(5): 634-663.

3. Boutroux L (1884) Sur la conservation des ferments alcooliques dans la nature. Annales des Sciences Naturelles, Série IV, Botanique 17: 145209.

4. Hansen EC (1890) Nouvelles recherches sur la circulation du Saccharomyces apiculatus dans la nature. Ann Sci Nat IV Ser Bot 11: 185-192.

5. Hartmann A, Rothballer M, Schmid M (2008) Lorenz Hiltner, a pioneer in rhizosphere microbial ecology and soil bacteriology research. Plant and Soil 312(1-2): 7-14.

6. Last FT (1955) Seasonal incidence of Sporobolomyces on cereal leaves. Transactions of the British Mycological Society 38(3): 221-239.

7. Zheng J, Yu X, Maninder M, Xu B (2018) Total phenolics and antioxidants profiles of commonly consumed edible flowers in China. International journal of food properties 21(1): 1524-1540. 